\title{
Combined QED and QCD Radiative Effects in Deep Inelastic Lepton-Proton Scattering: the Monte Carlo Generator DJANGO6
}

\author{
K. Charchuła, ${ }^{1}$ \\ Institute of Experimental Physics, Warsaw University, PL-00682 Warsaw, Poland, \\ G. A. Schuler, \\ Theory Division CERN, CH-1211 Geneva 23, Switzerland, \\ and \\ H. Spiesberger \\ Fakultät für Physik, Universität Bielefeld, D-33615 Bielefeld, Germany
}

\begin{abstract}
We describe the event generator DJANGO6, which simulates deep inelastic lepton-proton scattering including both QED and QCD radiative effects. DJANGO6 is an interface of the programs HERACLES 4.4 and LEPTO 6.1. The LUND-string fragmentation is used to obtain the complete hadronic final state. DJANGO6 is an update, which now also allows to use the matrix element options of LEPTO, which means that non-logarithmic corrections of $O\left(\alpha_{0}\right)$ can be taken into account.
\end{abstract}

CERN-TH.7133/94

January 1994

\footnotetext{
${ }^{1}$ Supported by the Polish State Committee for Scientific Research (grant No. 204209101).
} 


\section{Program Summary}

Program name:

Date of last modification:

Program language:

Number of program lines:

Other programs used:

External files needed:

Typical running time:

\section{DJANGO6}

25 September 1993

FORTRAN-77

about 14800 lines for HERACLES 4.4 and 5200 lines for LEPTO 6.1 and DJANGO6

JETSET 7.3, Lund Monte Carlo for jet fragmentation

Files for random number seeds and for information of the integration step are needed

Depending on the actual requirements such as parameters, requested contributions, kinematical limits; e.g. in the full DIS HERA kinematic region, the generation of 1000 radiative events with the $(\mathrm{ME}+\mathrm{PS})$ option of LEPTO, including hadronization, takes 80 seconds CPU on a VAX 9000-210 


\section{Introduction}

In the one-boson exchange approximation and taking into account higher-order QCD corrections only in the leading logarithmic approximation (LLA), the cross section for deep inelastic electron-proton scattering is described by two structure functions $F_{2}\left(x, Q^{2}\right)$ and $F_{3}\left(x, Q^{2}\right)$ :

$$
\frac{d^{2} \sigma}{d x d y}=\frac{2 \pi \alpha^{2}}{x y Q^{2}}\left[Y_{+} F_{2}\left(x, Q^{2}\right)+Y_{-} F_{3}\left(x, Q^{2}\right)\right]
$$

with

$$
Y_{ \pm}=1 \pm(1-y)^{2}
$$

$x, y$ and $Q^{2}$ are the usual kinematic variables for deep inelastic scattering. The structure functions are related to sums and differences of parton distributions $q_{f}\left(x, Q^{2}\right)$ :

$$
\begin{aligned}
& F_{2}\left(x, Q^{2}\right)=\sum_{f} A_{f}\left(Q^{2}\right)\left[q_{f}\left(x, Q^{2}\right)+\bar{q}_{f}\left(x, Q^{2}\right)\right], \\
& F_{3}\left(x, Q^{2}\right)=\sum_{f} B_{f}\left(Q^{2}\right)\left[q_{f}\left(x, Q^{2}\right)-\bar{q}_{f}\left(x, Q^{2}\right)\right],
\end{aligned}
$$

where $A_{f}$ and $B_{f}$ contain electroweak couplings and propagator terms. The scale dependence of $F_{2,3}$ is determined by the DGLAP evolution equations [1] and a possible contribution from the longitudinal structure function $F_{L}$ is neglected. The leading logarithms taken into account by these prescriptions emerge from gluon emission by the initial and final quark and from quark-antiquark pair production by gluons, evaluated in the LLA. These processes lead to the appearance of QCD cascades and are implemented in several Monte Carlo generators.

For the correct description of the differential cross section $d^{2} \sigma / d x d y$, as well as of details of the hadronic final state, it is, however, necessary to take into account also higherorder electroweak corrections and non-logarithmic QCD corrections. First, electroweak corrections, in particular hard photon radiation, lead to large effects and may strongly distort the kinematics of events for small $x$ and large $y$. Moreover, corrections from hard photon radiation strongly depend on experimental cuts. Secondly, it is well-known that $n$-jet cross sections in $e^{+} e^{-}$annihilation with $n \geq 3$ are not well described by leading logarithmic QCD cascades and matrix element calculations are needed. First measurements at HERA [2] indicate that this is also the case in deep inelastic ep scattering.

Monte Carlo programs for the simulation of $e p$ scattering at HERA including higherorder effects from either electroweak interactions or from QCD have been available for a long time and their descriptions are collected in [3]. There are also available two programs, DJANGO1.0 [4] and FRANEQ 2.0 [5], which interface electroweak radiative effects with parton cascades and fragmentation. These two programs use the parton shower model implemented in the obsolete version 5.2 of LEPTO [6] and thus include QCD effects in the LLA only.

In this note we present a technical description of the event generator DJANGO6. This program uses partly modified routines from LEPTO 6.1 [7] to simulate QCD effects and 
allows for generation of events according to QCD cascade models or QCD matrix elements. Matrix elements are available that describe effects from perturbative QCD to first order in $\alpha_{s}$ due to virtual gluons, real gluon emission and boson-gluon fusion. A combination of LLA QCD cascades with first-order matrix elements is possible. QCD cascades can be generated using either the parton shower model implemented in LEPTO 6.1 or the colour dipole model of ARIADNE [8]. Starting from an event defined at the parton level, i.e. having a final state that consists of quarks and gluons, one can generate the full hadronic final state with the help of JETSET 7.3 [9].

The process $l p \rightarrow l^{\prime} X$ including electroweak corrections of $O(\alpha)$ and the bremsstrahlung process $l p \rightarrow l^{\prime} \gamma X$ is simulated with the help of HERACLES, version $4.4[10,11]$.

In section 2 we provide a short discussion of QCD and QED radiative effects and describe the treatment of $O\left(\alpha \alpha_{s}\right)$ corrections as implemented in DJANGO6 as well as limitations of our approach. Section 3 is devoted to a detailed listing of technical details of the program.

\section{Physics and Monte Carlo implementation}

The radiative effects we are interested in can be classified in three categories: $i$ ) pure QCD corrections, ii) pure QED and weak corrections and iii) combined QCD-QED effects.

i) Corrections of $O\left(\alpha_{s}\right)$. The dominant parts of QCD corrections, i.e. the leading logarithms, are absorbed in the parton distribution functions leading to their scale dependence. These corrections are thus already included in Eq. (1), even resummed to all orders. Non-logarithmic $O\left(\alpha_{s}\right)$ corrections have to be taken into account explicitly. In the DIS scheme, these corrections show up as a non-zero longitudinal structure function $F_{L}$ and a small correction to $F_{3}$. Since the contribution from $F_{3}$ is small anyway at HERA energies, it is justified to neglect the $O\left(\alpha_{s}\right)$ corrections to $F_{3}$ and when restricting to parametrizations of parton distributions in the DIS scheme it is sufficient to add a contribution from $F_{L}$ to obtain the $O\left(\alpha_{s}\right)$ corrected cross section:

$$
\left.\frac{d^{2} \sigma}{d x d y}\right|^{O\left(\alpha_{*}\right)}=\left.\frac{d^{2} \sigma}{d x d y}\right|^{L L A}-\frac{2 \pi \alpha^{2}}{x y Q^{2}} y^{2} F_{L}\left(x, Q^{2}\right) .
$$

Here again, it is sufficiently accurate to include purely electromagnetic interactions, i.e. a contribution from $Z$ boson exchange to $F_{L}$ is ignored. $F_{L}$ can be defined in a way to absorb target mass effects and higher-twist contributions in addition to perturbative QCD (leading twist) contributions. The longitudinal structure function is taken into account in the calculation of the cross section by HERACLES.

The most important consequence of including $O\left(\alpha_{s}\right)$ corrections is, however, that they allow a more realistic and more detailed description of the hadranic final state: hard gluon emission and boson-gluon fusion give rise to events with two jets in addition to the proton remnant jet. Matrix elements for these processes to first order in $\alpha$, are available from LEPTO 6.1. We refer the reader to Ref. [7] for further details. 
ii) Electroweak corrections of $O(\alpha)$ consist of one-loop virtual corrections, which can be written in terms of form factors [12] modifying the coefficients $A_{f}$ and $B_{f}$ in Eq. (1) as well as of effects from real bremsstrahlung, i.e. radiative events $e p \rightarrow e \gamma X$. In the case of neutral current scattering, the radiative cross section can be separated into three parts [13], which are dominated by singularities due to $i$ ) emission of photons collinear with the incoming lepton, $i i$ ) emission of photons collinear with the scattered lepton, and iii) emission of hard photons shifting the momentum transfer $Q^{2}$ to small values, i.e. a part that is dominated by the Coulomb singularity.

Version 4.4 of HERACLES includes $O(\alpha)$ QED corrections due to photon radiation from both the lepton and the quark as well as the complete one-loop virtual corrections. The structure of the program allows a separate treatment of the nonradiative cross section (comprising the Born term, soft bremsstrahlung and virtual corrections) and the radiative cross section. The radiative cross section for the neutral current is separated into three channels associated with the three types of singularities described above. The separation into these three radiative channels is obtained on the basis of a partial fractioning of the differential cross section into corresponding pole terms. Note that the interpretation of the channels in terms of initial- or final-state radiation is meaningful only in the leading logarithmic approximation. Beyond the LLA, the separation is not unique. Note in particular that this separation is not a separation of the phase space for photon emission and that each channel is defined in the whole phase space.

HERACLES as well as DJANGO6 are also enabled for the simulation of charged current scattering. Here, besides the non-radiative channel, only one channel for the radiative process is activated at present.

No attempt was made yet to implement $O(\alpha)$ corrections due to (virtual and real) radiation of photons from the initial or scattered quark in DJANGO6, although these corrections can be taken into account in HERACLES. A consistent subtraction of quark mass singularities and matching to QCD cascades would be required. The observable effect of quarkonic radiation on the total deep inelastic cross section has been shown to be small at HERA energies [14].

iii) Finally, there are combined QCD-QED effects of order $O\left(\alpha \alpha_{s}\right)$. These corrections can be factorized in LLA. This is the basic property of higher-order corrections, which allows for a step-by-step procedure where the QED and QCD effects are described in separate programs. A more complete treatment would require the calculation of the combined $O\left(\alpha \alpha_{0}\right)$ matrix elements for

$$
\begin{aligned}
& e+q \rightarrow e+q+\gamma+g, \\
& e+g \rightarrow e+q+\bar{q}+\gamma .
\end{aligned}
$$

Since this is not yet done, non-logarithmic corrections of order $O\left(\alpha \alpha_{s}\right)$ are not included in DJANGO6.

The implementation of $O\left(\alpha \alpha_{s}\right)$ effects is described in more detail in the following. 


\subsection{Radiative Scattering}

The treatment of the non-radiative part of the cross section is straightforward: the Monte Carlo program HERACLES is used to generate events at the parton level, which in turn are fragmented and hadronized by DJANGO6 using routines from LEPTO 6.1 and JETSET 7.3. Therefore, in the following we only consider the radiative process

$$
e(l)+p(p) \rightarrow e^{\prime}\left(l^{\prime}\right)+\gamma(k)+X\left(p_{X}\right) .
$$

The particle momenta are given in parentheses. We define the standard DIS variables (so-called lepton variables) as the ones given by the inclusive lepton measurement, i.e.

$$
\begin{gathered}
q_{l}=l-l^{\prime}, \quad Q_{l}^{2}=-q_{l}^{2}, \\
x_{l}=\frac{Q_{l}^{2}}{2 p \cdot q_{l}}, \quad y_{l}=\frac{p \cdot q_{l}}{p \cdot l}, \quad W^{2}=\left(p+q_{l}\right)^{2}=\left(p_{X}+k\right)^{2} .
\end{gathered}
$$

The invariant mass $W$ includes the contribution of the radiated photon. At given squared centre-of-mass energy $s=(p+l)^{2}$, only two variables in (7) are independent by virtue of the relations

$$
Q_{l}^{2}=x_{l} y_{l} s, \quad W^{2}=\left(1-x_{l}\right) y_{l} s+m_{p}^{2} .
$$

The kinematics can also be defined through the hadronic final state. Provided the photon in (6) can be identified, one can actually measure the hadron variables:

$$
\begin{gathered}
q_{h}=p_{X}-p, \quad Q_{h}^{2}=-q_{h}^{2}, \\
x_{h}=\frac{Q_{h}^{2}}{2 p \cdot q_{h}}, \quad y_{h}=\frac{p \cdot q_{h}}{p \cdot l}, \quad W_{h}^{2}=\left(p+q_{h}\right)^{2}=p_{X}^{2} .
\end{gathered}
$$

Note that the variable $y_{h}$ is defined in terms of $p \cdot l$ rather than in terms of $p \cdot(l-k)$. The hadron variables obey relations in analogy to $(8)$ :

$$
Q_{h}^{2}=x_{h} y_{h} s, \quad W_{h}^{2}=\left(1-x_{h}\right) y_{h} s+m_{p}^{2} .
$$

In HERACLES, cuts can be imposed on the lepton variables $x_{l}, y_{l}, Q_{l}^{2}$, and, in addition, on the mass of the hadronic final state $W_{h}$.

\subsection{Factorization}

We use HERACLES to generate the variables for the semi-inclusive reaction (6). The momenta $l$ and $p$ are input quantities, thus $k$ and $l^{\prime}$ are the output of HERACLES. We then classify a given event according to the channels of HERACLES as being either nonradiative, an event with leptonic initial-state radiation, an event with leptonic final-state radiation, or a Compton event (cf. [13]). For radiative events we then define a rescaled Born-term-like process so that in any case we are left with

$$
E(L)+p(p) \rightarrow E^{\prime}\left(L^{\prime}\right)+X\left(p_{X}\right) .
$$

In general both $E$ and $E^{\prime}$ will be virtual particles. Since we restrict our considerations to $O\left(\alpha^{3}\right)$, only one of them will be virtual. 


\subsubsection{Initial state radiation}

In case the event is classified as resulting from leptonic initial state radiation we imagine the two-step process

$$
\begin{aligned}
e(l) & \rightarrow \tilde{e}(\tilde{l})+\gamma(k), \\
\tilde{e}(\tilde{l})+p(p) & \rightarrow e^{\prime}\left(l^{\prime}\right)+X\left(p_{\boldsymbol{X}}\right) ;
\end{aligned}
$$

thus $E(L)=\tilde{e}(\tilde{l})$ and $E^{\prime}\left(L^{\prime}\right)=e^{\prime}\left(l^{\prime}\right)$. Here $\tilde{l}=l-k$ and we define DIS variables for the $\tilde{e} p$ subprocess

$$
\begin{array}{rll}
\tilde{s} & =(\tilde{l}+p)^{2} & =s-2 k \cdot(l+p), \\
\tilde{Q}^{2} & =-\tilde{q}^{2} & \equiv-\left(\tilde{l}-l^{\prime}\right)^{2}, \\
\tilde{x} & =\frac{\tilde{Q}^{2}}{2 p \cdot \bar{q}} \quad=\frac{\tilde{Q}^{2}}{y s-2 p \cdot k}, \\
\tilde{y} & =\frac{\tilde{Q}^{2}}{\tilde{x} \tilde{s}}, & \\
\tilde{W}^{2} & =(p+\tilde{q})^{2}=(1-\tilde{x}) \tilde{y} \bar{s}+m_{p}^{2} .
\end{array}
$$

Note that the tilde variables coincide with the hadron variables (9) except for $s$ and $y$.

\subsubsection{Final state radiation}

A similar rescaling of variables is performed for events with leptonic final state radiation, now imagining the process as a hard scattering followed by a decay:

$$
\begin{aligned}
e(l)+p(p) & \rightarrow e^{\prime}\left(\tilde{l}^{\prime}\right)+X\left(p_{\boldsymbol{X}}\right), \\
\tilde{e}^{\prime}\left(\bar{l}^{\prime}\right) & \rightarrow e^{\prime}\left(l^{\prime}\right)+\gamma(k),
\end{aligned}
$$

where $\bar{l}^{\prime}=l^{\prime}+k$ and

$$
\begin{aligned}
\tilde{s} & =s \\
\tilde{Q}^{2} & =-\tilde{q}^{2} \quad \equiv-\left(l-\tilde{l}^{\prime}\right)^{2} \\
\tilde{x} & =\frac{\tilde{Q}^{2}}{2 p \cdot \tilde{q}}=\frac{\tilde{Q}^{2}}{y s-2 p \cdot k}, \\
\tilde{y} & =\frac{\tilde{Q}^{2}}{\tilde{x} \tilde{s}} .
\end{aligned}
$$

\subsubsection{Compton events}

Events from the Compton channel are characterized by typically small $Q^{2}$ and small $W_{h}$. For this reason they are not fragmented, and no hadronic final state is generated for them. 
The number of Compton events is small, typically a fraction of a per cent if a cut on the mass of the hadronic final state of $W_{h} \geq W_{h}^{\min }$ with $W_{h}^{\min } \simeq 2 \mathrm{GeV}$ is imposed.

For the reduced process (11) we then apply QCD corrections and the final partons and the proton remnants fragment using the LUND string fragmentation.

\subsection{Limitations of the approach}

DJANGO6 includes the most important corrections of order $\alpha, \alpha_{s}$, as well as combined QED-QCD effects of order $\alpha \alpha_{s}$. With respect to electroweak corrections, non-logarithmic corrections described by photon emission from quarks and box diagrams have not been taken into account. These contributions have been discussed in [15] and reach the level of a per cent only at very high momentum transfers $Q^{2}$. Although there is no physical interpretation of the separate channels of HERACLES beyond the leading logarithms, nevertheless, the cross section for $e p \rightarrow e X(\gamma)$ simulated by HERACLES includes both logarithmic and non-logarithmic leptonic corrections correctly to order $O(\alpha)$.

The calculation of the total cross section by HERACLES is not restricted to the use of parton model expressions for the structure functions. In particular, the contribution from the longitudinal structure function can be included correctly. In the event simulation, however, which is based on the parton model, the effect from $F_{L}$ can be treated only approximately. The contribution from $F_{L}$ is treated as if it gave rise to a hard scattering process involving in any case an incoming quark. The flavour of this quark is determined according to the flavour composition of $F_{2}$.

If the matrix element option is chosen, also non-logarithmic QCD corrections of order $O\left(\alpha_{0}\right)$ are correctly taken into account in our approach. The description of combined QED-QCD corrections of order $\alpha \alpha_{s}$ is based on the factorization property, which works in the leading logarithmic approximation. The precision that can be reached with this approach is most probably sufficient for HERA, except for rare processes with more than 2 jets in the final state (in addition to the remnant jet) and in the very low $x$ region where logarithms $\ln 1 / x$ have to be resummed.

According to the basic parton model assumption, the algorithms used in DJANGO6 are applicable only in phase space regions where the momentum transfer $Q_{h}^{2}$ and the final state hadronic mass $W_{h}$ are not too small. Therefore, default values of $Q_{\min }^{2}=4 \mathrm{GeV}^{2}$ and $W_{h, \min }=5 \mathrm{GeV}$ are used in DJANGO6. In order to avoid large virtual QED corrections, which could lead to a negative non-radiative cross section, the user should take care that $x_{l}$ and $y_{l}$ are restricted to the region

$$
y_{l}\left(1-x_{l}\right)^{2} \geq 0.004
$$

This excludes the region of very small $y_{l}$ and/or large $x_{l}$ where higher-order QED corrections (e.g. two- $\gamma$-radiation), which are not included in HERACLES, are important. From the experimental point of view this does not pose a severe restriction.

In HERACLES, much effort has been made to treat correctly small kinematic variables appearing, for instance, for collinear emission of photons. The precision of the algorithm 
has been checked by comparing it with other programs for the calculation of radiative corrections [15]. It has been shown that results from different programs agree usually at the per mille level and the agreement is better than $1 \%$ throughout the phase space relevant for HERA.

For events with a small hadronic mass of a few $\mathrm{GeV}$, round-off errors in DJANGO6 may lead to a small mismatch between energy or momentum of the initial state particles and the final state. For $W_{h}>5 \mathrm{GeV}$, only a few per mille of generated events have an energy in the final state that differs by more than $1 \%$ from the energy of the incoming particles. In view of the energy resolution of the HERA experiments $H 1$ and ZEUS, this will not be a severe problem in applications and work is in progress to improve the algorithm in this respect.

\section{Technical description}

The performance of DJANGO6 is determined by the structure of HERACLES. Inside DJANGO6 there are calls to routines calculating QED corrections, simulating QCD effects that determine the final state on the parton level, and in the end performing the hadronization. The user can communicate with the program by setting initial conditions (through input flags) and through a user analysis routine. The latter routine allows for $i$ ) additional initialization before generation of events (for example booking of histograms), ii) analysis after an event has been accepted, and iii) a final call after completion of the event generation. In the following section, input flags will be documented while in section 3.2 the user analysis routine and common blocks used to store the output of the program will be described.

\subsection{Input to the program}

The input to DJANGO6 consists of two parts: the first part deals with HERACLES options and the second one contains DJANGO6 input flags. The two parts are separated by the code word 'START' in the sequence of input items. The format for the input in the two parts is the same as for HERACLES, i.e. an option card defining the expected information (FORMAT (A10)) is followed by one card containing the appropriate data (format-free $\operatorname{READ}(5, *))$. All data items except 'START' (in the HERACLES part) and 'CONTINUE' (in the DJANGO6 part) are optional and are set to default values if they are not given as input. The sequence of different options within the two parts does not matter, only the 'CONTINUE' option triggering the actual operation of the program has to be the last one.

In the following we describe the possible input options and the corresponding data expected by the program both for the HERACLES and the DJANGO6 part. Default values are given in parentheses. For additional information concerning options of LEPTO 6.1 and JETSET 7.3 we refer to the corresponding manuals $[7,9]$. 


\subsubsection{HERACLES part}

- 'TITLE' data: user-defined heading of the first output page.

- 'EL-BEAM'

data: EELE, POLARI, LLEPT

quantities defining the properties of the electron beam;

EELE = energy of the electron beam in $\mathrm{GeV}(\mathrm{D}=30$.);

POLARI = degree of beam polarization $(\mathrm{D}=0),.-1 \leq P O L A R I \leq 1$;

LLEPT = lepton charge $(\mathrm{D}=-1)$,

$=-1$, electron beam,

$=+1$, positron beam.

- 'PR-BEAM'

data: EPRO

properties of the proton beam;

EPRO = energy of the proton beam in $\mathrm{GeV}(\mathrm{D}=820)$.

- 'KINEM-CUTS'

data: ICUT, XMIN, XMAX, YMIN, YMAX, Q2MIN, WMIN

definition of kinematical cuts to be applied;

ICUT $=1:$ cuts in $x_{l}$ and lower cut in $Q_{l}^{2}$ (Q2MIN in $\mathrm{GeV}^{2}$ ), cuts in $y_{l}$ are ignored,

$=2:$ cuts in $x_{l}$, lower cut in $Q_{l}^{2}$ and lower cut in the hadronic mass $W_{h}$ (WMIN in $\mathrm{GeV}$ ), cuts in $y_{l}$ are ignored,

$=3:$ cuts in $x_{l}, y_{l}, Q_{l}^{2}$ and $W_{h}$.

Default: ICUT $=3$. The final definition of cuts according to the most restrictive conditions is performed in the subroutine HSPRLG.

- 'EGAM-MIN'

data: EGMIN

definition of a lower cut-off energy for bremsstrahlung photons (in $\mathrm{GeV}$ );

EGMIN $=0.0:$ Both radiative and non-radiative events are generated. The separation of the cross section into non-radiative and radiative parts with the help of a soft-photon cut-off is determined internally as a function of $x$ and $y$. This option has to be used if integration/sampling of both radiative and non-radiative channels is requested. 
EGMIN > 0.0: Only hard-photon bremsstrahlung (with energy above EGMIN) is considered for event sampling (in this case the data items ISNC2 and ISCC2 from input options 'SAM-OPT-NC' and 'SAM-OPT-CC' are ignored!).

Default: $\mathrm{EGMIN}=0$.

- 'THMIN-QRAD'

not available in DJANGO6.

- 'gSW-Param'

data: LPARIN (1:11)

monitoring the definition of electroweak parameters and the inclusion of different virtual corrections;

LPARIN $(1)=1: \quad$ electroweak parameters set with fixed values for the boson masses $M_{W}, M_{Z}$,

$=2: \quad$ electroweak parameters calculated from fixed $M_{Z}, G_{\mu}$.

Default: LPARIN(1) $=2$. The definition of boson and fermion masses, as far as they are not input from code word 'GSW-MASS' and the calculation of coupling constants, is done in the subroutine HSSETP.

LPARIN (2) $=0$ : only Born cross section without electromagnetic or weak corrections is integrated/sampled,

$=1$ : Born cross section including corrections as determined by the values of LPARIN(3) - LPARIN(11).

Default: LPARIN(2) $=1$.

The following options define the corresponding corrections to be included in the actual calculation $(0 / 1=$ no/yes $)$ :

LPARIN (3) $(\mathrm{D}=3)$ : $\quad$ flag for inclusion of higher-order contributions:

$=0:$ no higher-order corrections,

$\geq 1:$ terms of $\mathcal{O}\left(\alpha^{2} m_{t}^{4}\right)$ included,

$\geq 2:$ terms of $\mathcal{O}\left(\alpha \alpha_{s} m_{t}^{2}\right)$ included,

$\geq 3$ : running $\alpha\left(Q^{2}\right)$ is used for leptonic corrections;

LPARIN (4) $(\mathrm{D}=1)$ : leptonic QED corrections;

LPARIN (5) $(\mathrm{D}=0)$ : quarkonic QED corrections (not active in DJANGO6);

LPARIN (6) $(D=0)$ : lepton-quark interference (not active in DJANGO6);

LPARIN (7) $(D=2)$ : fermionic contributions to the photon self energy $\Sigma^{\gamma}$ :

$=0:$ not included,

$=1:$ parametrization with the help of quark masses, 
$=2:$ parametrization from $[16]$;

LPARIN ( 8$)(\mathrm{D}=0)$ : fermionic contribution to the $\gamma-Z$ mixing;

LPARIN (9) $(\mathrm{D}=0)$ : fermionic contribution to the self energy of the $Z$ boson;

LPARIN (10) $(D=0)$ : fermionic contribution to the self energy of the $W$ boson;

LPARIN (11) $(D=0)$ : purely weak contributions to the self energies, vertex corrections and boxes.

- 'GSW-MASS'

data: MW, MZ, MH, MT

the electroweak mass parameters: $W$ and $Z$ boson masses, Higgs mass and the top-quark mass (in GeV). The value given for MW is only used if at the same time $\operatorname{LPARIN}(1)=1$. Otherwise $M W$ is calculated in the program from the $\mu$ decay constant.

- 'STRUCTFUNC'

data: ISTRFC

defines the parametrization of parton densities or structure functions applied in the calculation. The input is defined by

ISTRFC = ILQMOD*100000 + ILIB*10000 + ICODE.

ILQMOD encodes options for the treatment of structure functions in the low $Q^{2}$ region, ILIB defines a library of subroutines to be used for the calculation of parton distribution or structure functions and the corresponding parametrization is specified by ICODE. The meaning of ICODE depends on the value of ILIB. The allowed values are as follows:

ILIB $=1:$ to choose parton distribution functions from PYSTFU. The subroutine PYSTFU has been taken from LEPTO 6.1 [7] and modified for the use in HERACLES;

$$
\begin{aligned}
\text { ICODE } & =0: \text { Simple scaling distributions, } \\
& =1: \text { Eichten et al., set } 1 \text { of Ref. [17], } \\
& =2: \text { Eichten et al., set } 2 \text { of Ref. [17], } \\
& =3: \text { Duke/Owens, set } 1 \text { of Ref. [18], } \\
& =4: \text { Duke/Owens, set } 2 \text { of Ref. [18], } \\
& =5: \text { Morfin/Tung, set S1 of Ref. [19], } \\
& =6: \text { Morfin/Tung, set B1 of Ref. [19], } \\
& =7: \text { Morfin/Tung, set B2 of Ref. [19], } \\
& =8: \text { Morfin/Tung, set E of Ref. [19], } \\
& =9: \text { Glück, Reya and Vogt, LO [20], } \\
& =10: \text { Glück, Reya and Vogt, HO [20]; }
\end{aligned}
$$


ILIB $=2$ : to use PDFLIB [21]. Calls are performed via PYSTFU, but the corresponding line in the source code has to be enabled and the library to be provided by the user;

\section{ICODE = VALUE(1), the identification code for the parametrization} of parton distribution functions for PDFLIB ('MODE');

ILIB $=3:$ to use PAKPDF [22]. Again, calls are performed via PYSTFU;

$$
\begin{aligned}
I C O D E= & \text { IPAR } * 100+\text { ISET, the identification code for use of the } \\
& \text { library PAKPDF; }
\end{aligned}
$$

$$
\begin{array}{ll}
\text { ILQMOD }=0: & \text { use unmodified parton distribution functions for all } Q^{2} \\
\text { ILQMOD }=1: & \begin{array}{l}
\text { apply exponential low- } Q^{2} \text { suppression factor to parton distribu- } \\
\text { tion functions }[23] .
\end{array}
\end{array}
$$

The default values are ILQMOD $=1$ (exponential low $Q^{2}$ suppression), ILIB $=1$ (PDF's from PYSTFU) and ICODE $=9$ (Glück, Reya, Vogt, LO), i.e. ISTRFC $=110009$.

- 'FLONG'

data: IFLOPT

for the inclusion of the longitudinal structure function $F_{L}$. The definition of IFLOPT coincides with the definition of LST(11) of LEPTO 6.1 [7]:

IFLOPT $=$ LQCD + LTM*10 + LHT $* 100$

and LQCD, LTM and LHT specify whether QCD contributions, target mass effects and higher twist should be included. See the manual of LEPTO 6.1 [7] for details. HERACLES calls modified routines from LEPTO 6.1 to calculate $F_{L}$ either eventby-event when LQCD $=2$ or LTM $=2$ (time-consuming) or by using a grid and linear interpolation for $L Q C D=1$ or $L T M=1$.

For the calculation of radiative corrections, $F_{L}$ is needed for small $Q^{2}$, also in the limit $Q^{2} \rightarrow 0$. The prescription for $F_{L}\left(Q^{2} \rightarrow 0\right)$ is determined by ILQMOD. In the interface with DJANGO6 this means that $F_{L}$ is calculated from perturbative QCD. For $Q^{2}$ values smaller than $Q_{0}^{2}=6 \mathrm{GeV}^{2}, F_{L}$ is assumed to be independent of $Q^{2}$ and its value is given by $F_{L}\left(Q^{2}=Q_{0}^{2}\right)$.

The default value is IFLOPT $=0$.

- 'ALFAS'

data: MST111, MST115, PAR111, PAR112

information defining the value of $\alpha$, needed for the inclusion of $F_{L} ; \alpha$, enters the calculation of $F_{L}$ and is evaluated in the routine ULALPS from JETSET 7.3. The meaning of the input data corresponds to the parameters MSTU(111), MSTU(115), PARU (111), PARU(112) of JETSET 7.3 and is as follows:

MST111 : Order of $\alpha_{s}$ evaluation in ULALPS:

$=0$ : fixed $\alpha_{\text {a }}$ at the value of PAR111, 
$=1$ : first-order running $\alpha_{s}$,

$=2$ : second-order running $\alpha_{\theta}$,

$(\mathrm{D}=1)$

MST115: $\quad$ Treatment of the $\alpha_{1}$ singularity at $Q^{2} \rightarrow 0$ :

$=0$ : allows it to diverge like $1 / \ln \left(Q^{2} / \Lambda^{2}\right)$,

$=1:$ softens the divergence to $1 / \ln \left(1+Q^{2} / \Lambda^{2}\right)$,

$=2$ : freezes $Q^{2}$ evolution below $Q_{0}^{2}=4 \mathrm{GeV}^{2}$,

$(\mathrm{D}=0)$;

PAR111: $\quad$ The fixed value of $\alpha$, assumed in ULALPS when MST111 $=0$ $(\mathrm{D}=0.20)$;

PAR112: $\quad \Lambda$ used in running $\alpha_{\mathrm{s}}$ in ULALPS $(\mathrm{D}=0.25)$.

- 'NFLAVORS'

data: NPYMIN, NPYMAX

minimal and maximal number of flavours to be included in the cross section. The flavours are counted from 1 to 6 in the following order: $d, u, s, c, b$, and $t$. Defaults are NPYMIN $=1$ and NPYMAX $=6$.

- 'INT-ONLY'

data: INTOPT

A negative value for INTOPT suppresses the call to subroutines needed for the preparation of the sampling steps. Only integration of the differential cross section is performed in this case $(D=0)$.

- 'INT-OPT-NC'

data: INC2, INC31, INC32, INC33, INC34

defines the contribution(s) to neutral current interactions for which the integrated cross section is asked to be calculated in order to prepare the sampling procedure (including estimation of local maxima of the actual distribution function if INTOPT $\geq 0$ ):

INC2 : $\quad$ Integration for the non-radiative contribution (Born term including virtual and soft corrections); integration by Gaussian quadrature, NAGLIB routine DO1FCF,

INC31 < 100: number of iterations for integration of the contribution from initial state leptonic bremsstrahlung by VEGAS,

INC31 > 100: (INC31-100) iterations by VEGAS1; here the iteration procedure is restarted to estimate the integral; the grid structure is used from the previous run, but estimates of the integral and its error accumulated in the previous run(s) are discarded. The necessary information is read from an external file that had to be defined before by a run with INCx $<100$,

INC31 > 200: (INC31-200) iterations by VEGAS2: the iteration procedure is restarted using both the grid information and the accumulated estimates of the integral and its error from the previous run(s); 
INC32 : monitoring the integration of final state leptonic bremsstrahlung with the same conventions as for INC31;

INC33 : monitoring the integration of the Compton contribution with the same conventions as for INC31;

INC34 : monitoring the integration of the contribution from radiation from the quark line with conventions as for INC31;

Defaults are INC2 $=\mathrm{INC} 31=\mathrm{INC} 32=\mathrm{INC} 33=\mathrm{INC} 34=0$, no integration

- 'INT-OPT-CC'

data: ICC2, ICC31, ICC 32, ICC 33

same as 'INT-OPT-NC' but for the charged current interaction:

ICC2 : Integration for the non-radiative contribution (Born term with virtual and soft corrections);

ICC31 : Integration of the contribution from initial state leptonic bremsstrahlung by VEGAS;

ICC32 : Integration of the contribution from initial state quarkonic radiation (not yet active: set to 0 );

ICC33 : Integration of the contribution from final state quarkonic radiation (not yet active: set to 0 ).

- 'INT-POINTS'

data: NPOVEG

upper limit for the number of integration points used by VEGAS $(D=1000)$.

- 'SAM-OPT-NC'

data: ISNC2, ISNC31, ISNC32, ISNC33, ISNC34

monitoring the inclusion of individual contributions to the neutral current cross section for event sampling; default: ISNCx $=0$. A contribution is included if the corresponding option is set to 1 or 2 ; ISNCx $=2$ triggers continued sampling, i.e. information from a previous sampling run with ISNCX $\neq 0$ is expected:

ISNC2 : non-radiative contribution;

ISNC31 : initial state leptonic bremsstrahlung;

ISNC32: final state leptonic bremsstrahlung;

ISNC33 : Compton contribution;

ISNC34 : quarkonic radiation.

- 'SAM-OPT-CC'

data: ISCC2, ISCC31, ISCC32, ISCC33

same as 'SAM-OPT-NC' but for the charged current. For the physical content of the charged current radiative channels, see option 'INT-OPT-CC'. 
- 'RNDM-SEEDS'

data: ISDINP, ISDOUT

monitoring input/output of actual random seeds from/to unit LUNRND:

$$
\begin{array}{ll}
\text { ISDINP }=0 / 1: & \text { (no) input of seeds; } \\
\text { ISDOUT }=0 / 1: & \text { (no) } \text { output of seeds; }
\end{array}
$$

default: ISDINP $=$ ISDOUT $=0$.

- 'IOUNITS'

data: LUNOUT, LUNRND, LUNDAT, LUNPD6, LUNPD7

logical unit numbers for in- and output:

LUNOUT: for standard output $(\mathrm{D}=6)$;

LUNDAT: for in-/output of results from the integration step from/to an external file $(\mathrm{D}=11)$;

LUNRND: for in-/output of the random number status from/to an external file $(\mathrm{D}=10)$;

LUNPD6: not used;

LUNPD7: not used.

- 'START'

data: NEVENT

starts the execution of the main program;

NEVENT : number of requested events if any sampling option is activated $(\mathrm{D}=$ $1000)$.

\subsubsection{DJANGO6 part}

- 'OUT-LEP'

data: LST (4) (integer, $\mathrm{D}=1$ )

regulates which information is written onto the event record:

$$
\begin{aligned}
\operatorname{LST}(4)= & \mathrm{I}_{\text {lepton }}+10 * \mathrm{I}_{\text {shower }} ; \\
& \mathrm{I}_{\text {lepton }}=0 / 1 \text { inactive } / \text { active scattered electron; } \\
& \mathrm{I}_{\text {shower }}=0 / 1 \text { exclude/include intermediate partons. }
\end{aligned}
$$

- 'FRAME'

data: $\operatorname{LST}(5)$ (integer, $\mathrm{D}=3$ )

choice of frame for the event:

LST (5) = 1: hadronic c.m.s. frame, $z$-axis along boson,

$=2: e p$ c.m.s. frame, $z$-axis along lepton, 
$=3: e p$ lab system, $z$-axis along lepton,

$=4$ : ep lab system, $z$-axis along exchanged boson.

- 'FRAG'

data: LST ( 7 ) (integer, $\mathrm{D}=1$ )

LST(7) $=0$ : event generation at the parton level, no hadronization,

$=1$ : event generation including hadronization and decays of unstable particles.

- 'CASCADES'

data: $\operatorname{LST}(8)$ (integer, $D=12$ )

describes that part of the event simulation that is determined by perturbative QCD:

LST (8) $=0$ : no QCD effects, i.e. no gluon radiation or boson-gluon fusion, $=1$ : including QCD processes (gluon radiation and boson-gluon fusion) according to the first-order matrix elements,

$=2$ : QCD parton cascade evolution of initial and final quark,

= 3: QCD parton cascade evolution of initial quark only,

$=4:$ QCD parton cascade evolution of final quark only,

$=5: \mathrm{QCD}$ switched off, but target treatment exactly as in parton cascade case,

= 9: simulating QCD cascades in the colour dipole model as implemented in ARIADNE,

$=12-15$ : as $2-5$, but parton shower added to the event as obtained from first-order matrix elements (ME+PS).

- 'MAX-VIRT'

data: LST (9) (integer, $\mathrm{D}=5$ )

maximal virtuality in parton cascades (active only for LST $(8)=2-5$ ):

$$
\begin{aligned}
\operatorname{LST}(9) \quad & =1: Q^{2}, \\
& =2: W^{2}, \\
& =3: W \times Q \\
& =4: Q^{2} \times(1-x), \\
& =5: Q^{2} \times(1-x) \times \max (1, \ln (1 / x)), \\
& =9: W^{4 / 3}, \text { i.e. similar as in the colour dipole model. }
\end{aligned}
$$

- 'BARYon'

data: $\operatorname{LST}$ (14) (integer, $\mathrm{D}=1$ )

treatment of the target remnant (for more detailed information, see [7]):

LST(14) = 0: baryon production from remnant excluded, 
$=1$ : baryon production from remnant included,

$=2,3:$ as 1 but with different energy-momentum fractions distributed over remnant parts.

- 'KT-PARTON'

data: PARL (3) (real, $\mathrm{D}=0.44$ )

width of Gaussian primordial transverse momentum distribution (in $\mathrm{GeV}$ ).

- 'DIQUARK'

data: PARL (4) (real, $\mathrm{D}=0.75$ )

probability that a $u d$ diquark in target remnant has spin and isospin equal to zero, i.e. $\mathrm{I}=\mathrm{S}=0$.

- 'KT-REMNANT'

data: PARL (14) (real, D=0.44)

width of Gaussian $p_{T}$ when non-trivial target remnant is split into a particle and a jet (in $\mathrm{GeV}$ ).

- 'AR-REMNANT'

data: MSTA (30) (integer, $\mathrm{D}=1$ )

extendedness of initial partons in ARIADNE (see [8]);

MSTA (30) $=0$ : struck quark is point-like and proton remnant extended with $\mu=$ PARA (11) (= $1 \mathrm{GeV}$ by default), where $\mu$ is the inverse extension of the proton remnant,

$=1:$ as for 0 but $\mu=\operatorname{PARA}(11) /(1-x)$,

$=2$ : as for 1 but also struck quark is extended with $\mu=\sqrt{Q^{2}}$.

- 'CONTINUE'

continue execution of the main program, start event generation.

\subsection{Output and User's analysis}

Any user action is expected in the user-supplied subroutine HSUSER (ICALL , $X, Y, Q 2$ ). The routine allows for user action in three different phases of running DJANGO6:

- before generating events (ICALL=1),

- after each generated event (ICALL=2),

- after completion of event generation (ICALL=3).

The arguments $X, Y, Q 2$ correspond to the lepton kinematic variables of the actual event.

For each sampled event the information about the $e q \rightarrow e q(\gamma)$ subprocesses is transferred to HSUSER via the HERACLES common blocks /HEPEVT/, /HSIKP/ and /HSCHNN/ 
as described in [10]. The information about the hadronic final state is contained in the common block /LUJETS/ of JETSET 7.3 [9]. In case of an event with hard QED bremsstrahlung, the information about the radiated photon is found in /LUJETS/ right after the scattered electron, i.e. in line 5. Additional information is contained in the common blocks /LINTEG/ (of HERACLES) and /LEPTOU/ (of LEPTO). Below we describe those common blocks, which can be useful for the analysis of the event. For details about the content of the common block /LUJETS/, which contains the event record, we refer the reader to the manual of JETSET 7.3 [9].

\subsubsection{Common block LEPTOU}

COMMON /LEPTOU/ CUT (14), LST(40), PARL (30), X, Y, W2, Q2,U

Content: contains input switches (LST(1) - LST (20)) and input parameters (PARL (1) - PARL (20)) for LEPTO to specify physics, kinematic cuts and numerical procedures, as well as output flags (LST(21) - LST (40)) and output variables (PARL (21) - PARL (30)). The initialization of input switches and parameters is done either through input flags of DJANGO6 or in BLOCK DATA LEPTOD.

Parameters:

CUT(1) - CUT (14): lower and upper limits on $x_{l}, y_{l}, Q_{l}^{2}, W_{h}^{2}, \nu, E^{\prime}, \theta^{\prime}$; included for documentation purposes only (passed from HERACLES).

LST(1) : $\quad(D=2)$ not used;

LST (2) : $\quad(\mathrm{D}=3)$ scaled variables $(\bar{x}, \bar{y})$ passed via LEPTOU;

LST (3) : $\quad(\mathrm{D}=5)$ detailed output at all initialization calls;

LST (4) : $\quad(D=1)$ user input via code-word 'OUT-LEP';

LST (5) : $\quad(\mathrm{D}=3)$ user input via code-word 'FRAME';

LST (6) : $\quad(D=0)$ no rotation of the azimuthal angle, $\phi$, of the lepton scattering plane; random azimuthal orientation passed from HERACLES;

LST (7) : $\quad(\mathrm{D}=1)$ user input via code-word 'FRAG';

LST (8) : $\quad(\mathrm{D}=12)$ user input via code-word 'CASCADES';

LST (9) : $\quad(D=5)$ user input via code-word 'MAX-VIRT';

LST(10): $\quad(D=1)$ not used;

LST(11): $\quad(D=0)$ user input via code-word 'FLONG';

LST(12) : $\quad(D=6)$ NPYMAX, maximum flavour used in the sea structure function parametrizations, passed from HERACLES;

LST (13): $\quad(D=5)$ not used;

LST(14): $\quad(D=1)$ user input via code-word 'BARYON';

LST(15): $(D=7)$ choice of the parametrization for parton densities in the nucleon, NPYMOD, passed from HERACLES;

LST (16): $\quad(D=1)$ choice of the structure function library, passed from HERACLES;

LST (17): $\quad(D=1)$ varying energies of initial particles from event to event; 
LST(18): $\quad(\mathrm{D}=2)$ not used;

LST (19): $\quad(D=3)$ choice of grid for first-order QCD weights, suitable for ep collisions in HERA;

LST (20): $\quad(\mathrm{D}=0)$ not used;

LST(21): $\quad$ error flag, $=0$ for properly generated event;

LST (22): $\quad(D=1)$ information about chosen target nucleon in current event (proton);

LST (23): $\quad$ specifies simulated process;

$=2$ : weak charged current $(\mathrm{CC})$, i.e. $\mathrm{W}^{ \pm}$exchange;

$=4$ : neutral current (NC), i.e. $\gamma / Z^{0}$ exchange;

LST (24) : information about first-order QCD process in current event;

$=1: q$-event, i.e. no first-order QCD;

$=2$ : $q g$-event, i.e. gluon radiation in first-order $\mathrm{QCD}$;

$=3: q \bar{q}$-event, i.e. boson-gluon fusion in first-order QCD;

LST (25) : information about flavour of the struck quark in current event: $1=d, 2=u$, $3=s, 4=c, 5=b,-1=\bar{d},-2=\bar{u},-3=\bar{s},-4=\bar{c},-5=\bar{b}$;

LST (26) : entry line in event record of outgoing struck quark. In parton shower case, quark at boson vertex before final state shower;

LST(27) : signals split of non-trivial nucleon remnant, cf. LST(14);

$=0$ : no split, simple diquark or $\operatorname{LST}(14)=0$;

$=1$ : split into parton and particle, $q q+M$ or $q+B$, occurs when sea (anti)quark is removed through the interaction;

$=2$ : split into quark and diquark, $q+q q$, occurs when a gluon is removed;

LST(28): specifies the frame in which the current event is given with code as for LST (5);

LST (29): $\quad$ specifies azimuthal angle rotation with code as for LST(6);

$\operatorname{LST}(30)-\operatorname{LST}(40):(D=0)$ not used.

PARL (1) : $\quad(\mathrm{D}=1$.$) not used;$

PARL (2) : $(\mathrm{D}=1$.$) not used;$

PARL (3) : $\quad(D=0.44)$ user input via code-word 'KT-PARTON';

PARL (4) : $\quad(\mathrm{D}=0.75)$ user input via code-word 'DIQUARK';

PARL (5): $\sin ^{2} \theta_{W}$ (weak mixing angle), passed from HERACLES (SW2);

PARL (6) : polarization of lepton beam, passed from HERACLES (POLARI);

PARL (7) : not used;

PARL (8), PARL (9): $\left(\mathrm{D}=0.015,2\right.$.) minimum value of $y=m_{i j}^{2} / W^{2}$ and $m_{i j}$, where $m_{i j}$ is the invariant mass of any parton pair (including target remnant), used as cutoffs against divergences in first-order QCD matrix elements;

Remarks: PARL ( 8$)$ is the dominating cut at large energy $(W)$ whereas PARL (9) dominates at low energy. These are starting values when integrating first-order QCD matrix elements, but the effective cut used, PARL (27), is automatically increased in order that the QCD probabilities do not exceed unity for any values of $x$ and $W$;

PARL (10): not used; 
PARL (11): $(D=0.01)$ required relative accuracy for one-dimensional integration, used for first-order QCD matrix element weights and longitudinal structure function integrals;

PARL (12), PARL (13): ( $D=4,0.1)$ internal parameters used for adjustment of $y_{c u t}$ for integration of QCD matrix elements;

PARL (14): $\quad(D=0.44)$ user input via code-word 'KT-REMNANT';

PARL (15): $(\mathrm{D}=0.01)$ not used;

PARL (16): fine structure constant $\alpha$, passed from HERACLES (ALPHA);

PARL (17): weak Fermi constant $G_{F}$, passed from HERACLES (GF);

PARL (18): $\Delta r$ from radiative corrections, passed from HERACLES (DELTAR);

PARL (19): $\quad(D=0.03)$ scale $\kappa^{2}$ in $\mathrm{GeV}^{2}$ for higher twist correction;

PARL (20): $(\mathrm{D}=1$.) not used;

PARL (21): scaled $\bar{s}$;

PARL (22): $\quad$ scaled $\tilde{y} \tilde{s}\left(=y_{h} s_{h}\right)$;

PARL (23): total cross section in $p b$, passed from HERACLES (= SIGTOT*10 ${ }^{3}$ );

PARL (24) : Monte Carlo estimate of the cross section in picobarns associated with the generated event sample;

PARL (25): value of $\alpha_{s}$ in current event;

PARL (26): value of $\Lambda$ used in last structure function call, passed from HERACLES (filled only when LST $(16)=1$ );

PARL (27): present value of the $y$-cut for first-order QCD. Given by maximum of PARL (8) and PARL (9), but modified internally to prevent QCD weights exceeding unity.

PARL (28) - PARL (30) : not used;

$\mathrm{X}, \mathrm{Y}, \mathrm{W} 2, \mathrm{Q} 2, \mathrm{U}:$ rescaled variables $\bar{x}, \bar{y}, \tilde{W}^{2}, \tilde{Q}^{2}, \bar{\nu}$, see section 2.2.1. Except for $\bar{y}$, the rescaled variables are identical to the hadron variables, Eq. (9).

\subsubsection{Common block LINTEG}

COMMON /LINTEG/ NTOT, NPASS

Content: $\quad$ contains statistics of generated events:

Parameters:

NTOT : $\quad$ Total number of events generated by HERACLES;

NPASS : $\quad$ Number of events that have been hadronized completely;

Remarks: After normal completion of the program, NTOT and NPASS should be equal. NPASS is smaller than the total number of events generated by HERACLES when the program was unable to hadronize all events. The difference NTOT - NPASS is used to calculate the efficiency of the algorithm. The total cross section derived from HERACLES is corrected accordingly and written to PARL (24) in the common block /LEPTOU/. 


\subsubsection{Common block HSCHNN}

COMMON /HSCHNN/ ICHNN

Content: contains information on the origin of the event from the channels of HERACLES

Parameters:

ICHNN : $\quad$ flag of channel, passed from HERACLES:

$=1$ : non-radiative neutral current $(\mathrm{NC})$ event,

$=2$ : non-radiative charged current $(\mathrm{CC})$ event,

$=6:$ leptonic initial state photon radiation $(\mathrm{NC})$,

$=7$ : leptonic final state photon adiation (NC),

$=8$ : Compton event (NC),

$=12$ : radiative charged current event $(\mathrm{CC})$.

\subsection{Implementation}

\subsubsection{External files}

The program uses two external files:

unit LUNRND : file to store/read random number seeds (101 double-precision words on the IBM), see code word 'RNDM-SEEDS'. The user can initialize this file with a first run of the program with ISDINP $=0$ and ISDOUT $=1$;

unit LUNDAT : $\quad$ information from integration procedures (and potential previous event sampling), needed for (continued) event generation, see code word 'INT-OPT-NC'.

\subsubsection{External procedures required by the program}

The following external routines/packages are being used by DJANGO6:

D01FCF: integration routine from the NAGLIB library. A substitute for this routine is contained in the file DJHERAC.FOR (see next section);

JETSET 7.3: The LUND Monte Carlo for jet fragmentation [9];

ARIADNE 4: The LUND Monte Carlo for colour dipole cascade [8].

The user has to take care that JETSET 7.3 and ARIADNE 4 are available to the linker. In case ARIADNE is not going to be linked one needs to enable two dummy routines, ARINIT and AREXEC, contained in DJUSER.FOR (see next section). 


\subsubsection{Source code}

The official version of the program can be found on the DESY VAX directory:

$$
\text { DISK\$ZOF : [CHARCHULA . DJANGO] }
$$

or obtained from one of the authors via e-mail (addresses: charchula@vxdesy.desy.de or i02spiedsyibm.desy.de).

The source code of DJANGO6 has been divided into three parts:

DJHERAC.FOR: routines from HERACLES 4.4;

DJLEPTO.FOR: routines from LEPTO 6.1 used by DJANGO6 (some of them modified) and new routines of DJANGO6;

DJUSER.FOR: contains an example of the user routine HSUSER and two (commented out) dummy routines, ARINIT and AREXEC, used when ARIADNE is not going to be linked.

Furthermore, sample .com-files to run the program on a VAX/VMS are provided:

DJEXE.COM: an example batch file to make an *.EXE file;

DJRUN.COM: an example batch file to run the program;

DJINIT.IN: a file containing setting of input for DJANGO6.

Finally, the user can consult an information file DJANGO. INFO where updates of the program will be announced and there is a copy of the TEX file DJANGO.TEX containing this manual.

\section{Acknowledgements}

The authors thank G. Ingelman for providing the source of the LEPTO 6.1 routines and for the permission to use part of the LEPTO 6.1 manual in this document.

\section{References}

[1] V. N. Gribov and L. N. Lipatov, Sov. J. Nucl. Phys. 15 (1972) 438 and 675;

G. Altarelli and G. Parisi, Nucl. Phys. B126 (1977) 297;

Y. L. Dokshitser, Sov. Phys. JETP 46 (1977) 641.

[2] H1 Collab., DESY 92-162;

ZEUS Collab., DESY 93-068;

H1 Collab., DESY 93-137. 
[3] W. Buchmüller and G. Ingelman, eds., Proc. of the Workshop on Physics at HERA, DESY (1992), Vol. 3.

[4] G. A. Schuler and H. Spiesberger, in same Proc. as in ref. [3], Vol. 3, p. 1419.

[5] W. Placzek, in same Proc. as in ref. [3], Vol. 3, p. 1294.

[6] G. Ingelman, LEPTO version 5.2, unpublished program manual.

[7] G. Ingelman, LEPTO 6.1, in same Proc. as in ref. [3], Vol. 3, p. 1366.

[8] L. Lönnblad, Comput. Phys. Commun. 71 (1992) 15; L. Lönnblad, in same Proc. as in ref. [3], Vol. 3, p. 1440.

[9] T. Sjöstrand, Comput. Phys. Commun. 39 (1986) 347; T. Sjöstrand and M. Bengtsson, Comput. Phys. Commun. 43 (1987) 367; T. Sjöstrand, CERN-TH.6488/92.

[10] A. Kwiatkowski, H.-J. Möhring and H. Spiesberger, Comput. Phys. Commun. 69 (1992) 155.

[11] H. Spiesberger, HERACLES version 4.4, unpublished program manual (1993); version 4.1 is described in A. Kwiatkowski, H.-J. Möhring and H. Spiesberger, in same Proc. as in ref. [3], Vol. 3, p. 1294.

[12] W. Hollik et al., in same Proc. as in ref. [3], Vol. 2, p. 923.

[13] A. Kwiatkowski, H.-J. Möhring and H. Spiesberger, Z. Phys. C 50 (1991) 165.

[14] J. Kripfganz and H. Perlt, Z. Phys. 41 (1988) 319.

[15] H. Spiesberger et al. in same Proc. as in ref. [3], Vol. 2, p. 798.

[16] H. Burkhardt, F. Jegerlehner, G. Penso, and C. Verzegnassi, in: Polarization at LEP, CERN 88-06, Eds. G. Alexander et al., Vol. 1, p. 145.

[17] E. Eichten, I. Hinchliffe, K. Lane, and C. Quigg, Rev. Mod. Phys. 56 (1984) 579.

[18] D. W. Duke, J. F. Owens, Phys. Rev. D30 (1984) 49.

[19] J. G. Morfin and W. K. Tung, Z. Phys. C52 (1991) 13.

[20] M. Glück, E. Reya and A. Vogt, Z. Phys. C48 (1990) 471.

[21] H. Plothow-Besch, Comput. Phys. Commun. 75 (1993) 396.

[22] K. Charchula, Comput. Phys. Commun. 69 (1992) 360.

[23] N. Yu. Volkonsky and L. V. Prokhorov, Sov. J. Exp. Theor. Phys. Lett. 21 (1975) 389. 


\section{Appendix}

Below we show an example for DJANGO6 input cards and part of the output from the corresponding run of the program. The output shows only the structure of the first part where HERACLES, DJANGO6 and LEPTO 6.1 print their input and final settings of parameters. Then, three events generated in the non-radiative, the initial state radiation and the final state radiation channel for neutral current scattering are given.

(1) An example for input cards :

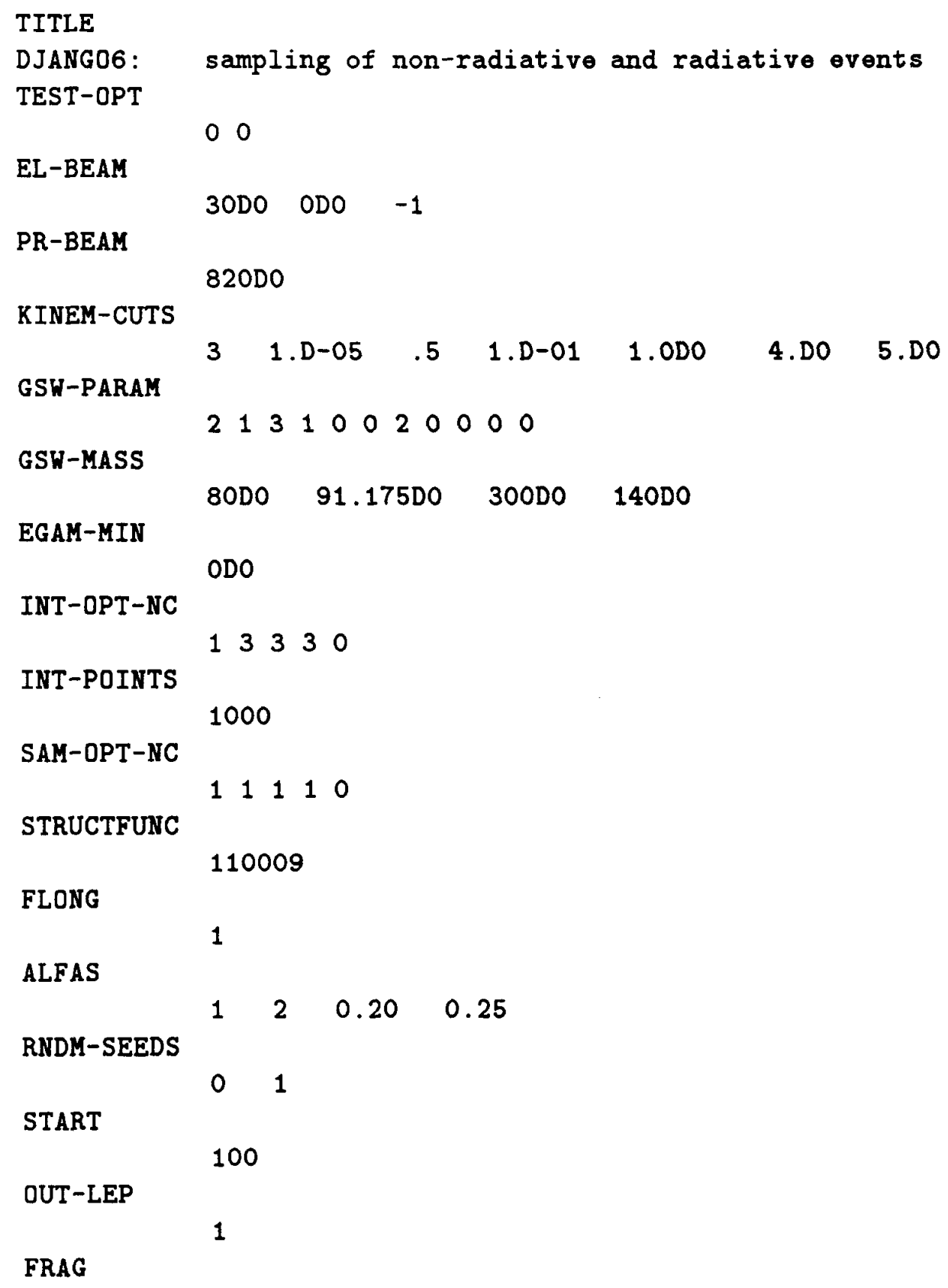

FRAG 
CONTINUE

/*

(2) Output listing:

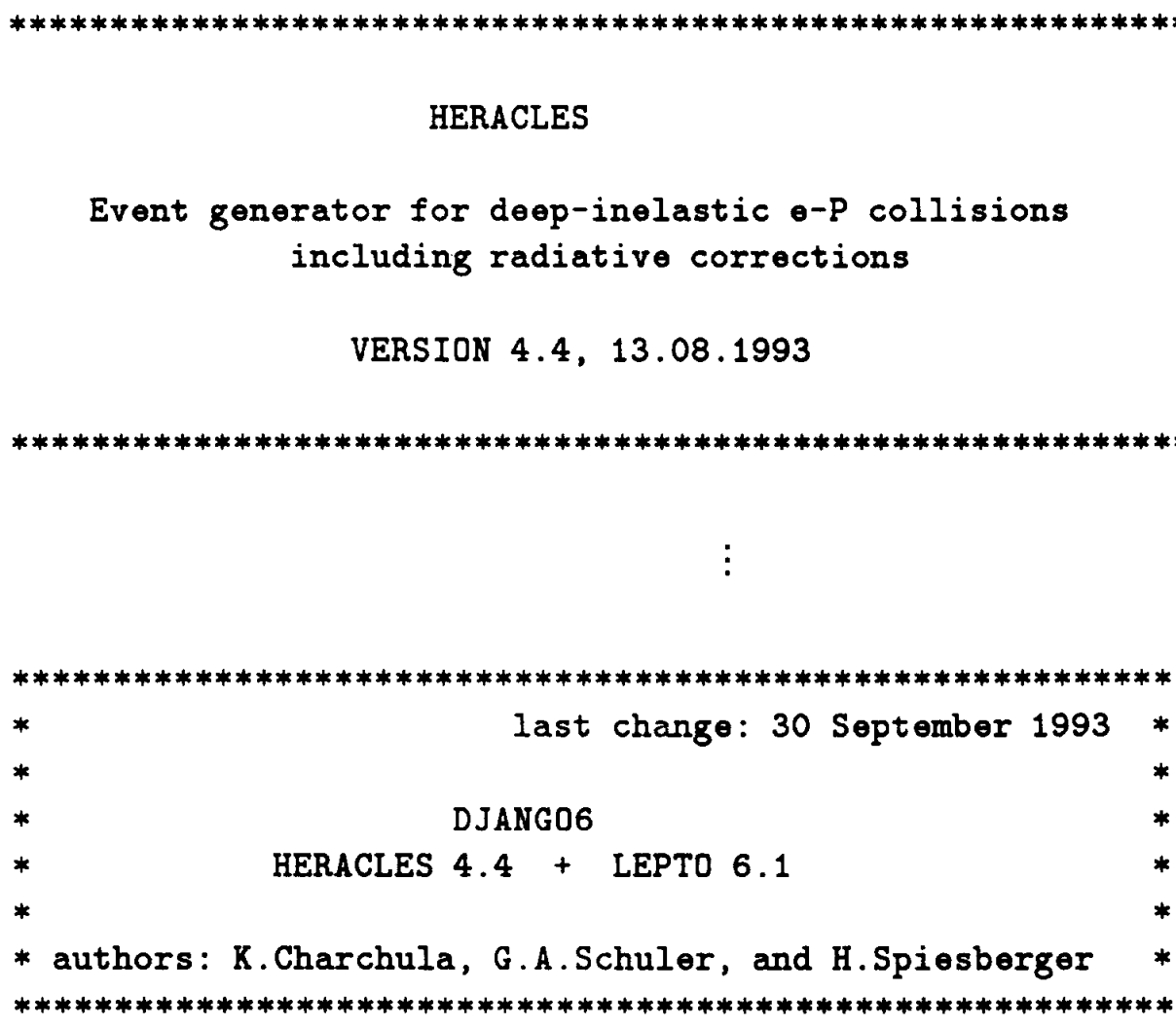

THE LUND MONTE CARLO FOR DEEP INELASTIC LEPTON-NUCLEON SCATTERING

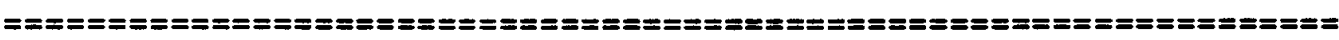
LEPTO version 6.1, May 4, 1992 
CROSS SECTIONS ACTUALLY APPLIED FOR SAMPLING (IN NANOBARN):

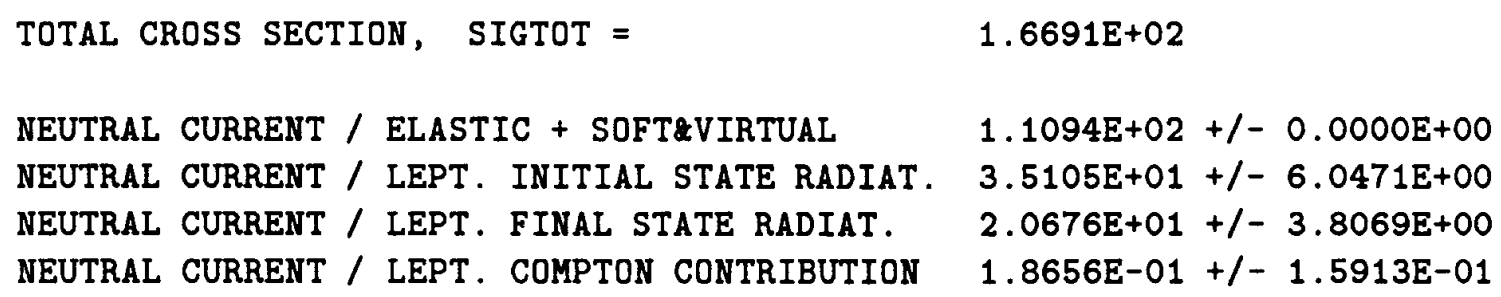

\begin{tabular}{|c|c|c|c|c|c|c|}
\hline CHANNEL = & $\mathrm{x}$ & $\mathbf{Y}$ & & & & \\
\hline leptonic: & $0.177 E-02$ & 0.50 & $8+00$ & 0.8 & $E+02$ & \\
\hline hadronic: & $0.177 \mathrm{E}-02$ & 0.50 & $8+00$ & 0.8 & $E+02$ & \\
\hline scaled: & $0.177 \mathrm{E}-02$ & 0.50 & $2+00$ & 0.8 & $E+02$ & \\
\hline$---\infty--$ & $\mathrm{Px}$ & Py & & $\mathrm{Pz}$ & E & M \\
\hline HS e-scat: & -4.8586 & -4.4657 & 14 & 1941 & 15.6531 & 0.0005 \\
\hline HS q-scat: & 4.8586 & 4.4657 & -804 & 1935 & 834.3464 & 222.1800 \\
\hline HS ga-rad: & 0.0000 & 0.0000 & & 0000 & 0.0000 & 0.0000 \\
\hline
\end{tabular}

Event listing (summary)

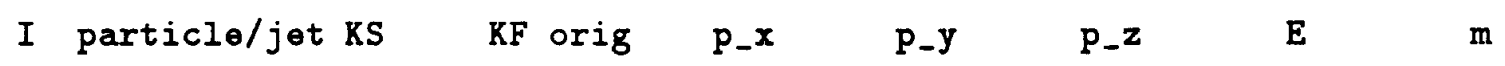

\begin{tabular}{|c|c|c|c|c|c|c|c|c|c|c|}
\hline & !e-! & & 21 & 11 & 0 & 0.000 & 0.000 & 30.000 & 30.000 & 0.00 \\
\hline 2 & $! p+!$ & & 21 & 2212 & 0 & 0.000 & 0.000 & -819.999 & 820.000 & 0. \\
\hline & !ZO! & & 21 & 23 & 1 & 4.859 & 4.466 & 15.806 & 14.347 & -9. \\
\hline & $\theta^{-}$ & & 1 & 11 & 1 & -4.859 & -4.466 & 14.194 & 15.653 & \\
\hline & $u$ & A & 2 & 2 & 0 & 5.144 & 4.976 & 14.227 & 15.926 & \\
\hline & ud_o & v & 1 & 2101 & 2 & -0.285 & -0.510 & -818.420 & 818.421 & 0 \\
\hline & & & um: & 0.00 & & 0.000 & 0.000 & -789.999 & 850.000 & 13.6 \\
\hline
\end{tabular}

\begin{tabular}{lccccc} 
CHANNEL $=$ & 6 & $\mathrm{X}$ & \multicolumn{4}{c}{$\mathrm{Q}$} & \\
leptonic: & $0.125 \mathrm{E}-03$ & $0.627 \mathrm{E}+00$ & $0.769 \mathrm{E}+01$ & \\
hadronic: & $0.264 \mathrm{E}-02$ & $0.126 \mathrm{E}-01$ & $0.328 \mathrm{E}+01$ & \\
scaled: & $0.264 \mathrm{E}-02$ & $0.327 \mathrm{E}-01$ & $0.328 \mathrm{E}+01$ & \\
\hline HS e-scat: & 1.2166 & -1.1786 & 11.1359 & 11.2640 & 0.0005 \\
HS q-scat: & -1.2275 & 1.2939 & -819.5574 & 820.3137 & 35.1716 \\
HS ga-rad: & 0.0109 & -0.1153 & 18.4220 & 18.4223 & 0.0000
\end{tabular}

Event listing (summary)

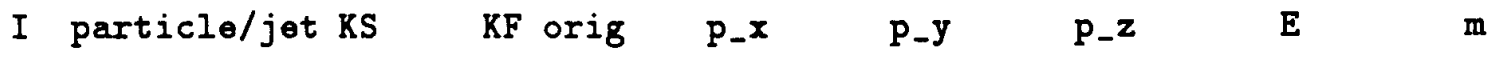




\begin{tabular}{|c|c|c|c|c|c|c|c|c|c|c|}
\hline 1 & $! \theta-!$ & & 21 & 11 & 0 & 0.000 & 0.000 & 30.000 & 30.000 & 0.001 \\
\hline 2 & $! p+!$ & & 21 & 2212 & 0 & 0.000 & 0.000 & -819.999 & 820.000 & 0.938 \\
\hline 3 & !zo! & & 21 & 23 & 1 & -1.227 & 1.294 & 0.442 & 0.314 & -1.811 \\
\hline 4 & $\theta-$ & & 1 & 11 & 1 & 1.217 & -1.179 & 11.136 & 11.264 & 0.001 \\
\hline 5 & gamma & & 1 & 22 & 1 & 0.011 & -0.115 & 18.422 & 18.422 & 0.000 \\
\hline 6 & $\mathbf{u}$ & A & 2 & 2 & 0 & -2.564 & -4.216 & -20.452 & 21.039 & 0.062 \\
\hline 7 & ud_o & v & 1 & 2101 & 2 & -0.156 & 0.062 & -249.936 & 249.937 & 0.57 \\
\hline 8 & $\mathrm{u}^{\sim}$ & A & 2 & -2 & 0 & 1.639 & 5.512 & -97.963 & 98.131 & 0.06 \\
\hline 9 & $\mathrm{u}$ & V & 1 & 2 & 2 & -0.146 & -0.064 & -451.382 & 451.382 & 0.06 \\
\hline & & & sum: & 0.00 & & 0.000 & 0.000 & -790.175 & 850.176 & 313.7 \\
\hline
\end{tabular}

\begin{tabular}{lccccr} 
CHANNEL $=$ & 7 & $\mathrm{X}$ & \multicolumn{4}{c}{$\mathrm{Q}$} & \multicolumn{2}{c}{$\mathrm{Q}$} & \\
leptonic: & $0.179 \mathrm{E}-03$ & $0.253 \mathrm{E}+00$ & $0.447 \mathrm{E}+01$ & \\
hadronic: & $0.185 \mathrm{E}-01$ & $0.272 \mathrm{E}-02$ & $0.495 \mathrm{E}+01$ & \\
scaled: & $0.185 \mathrm{E}-01$ & $0.272 \mathrm{E}-02$ & $0.495 \mathrm{E}+01$ & \\
\hline HS e-scat: & -1.7789 & 0.4197 & 22.3607 & 22.4353 & 0.0005 \\
HS q-scat: & 2.1378 & -0.6039 & -819.8752 & 820.0388 & 16.2295 \\
HS ga-rad: & -0.3589 & 0.1842 & 7.5151 & 7.5259 & 0.0000
\end{tabular}

Event listing (summary)

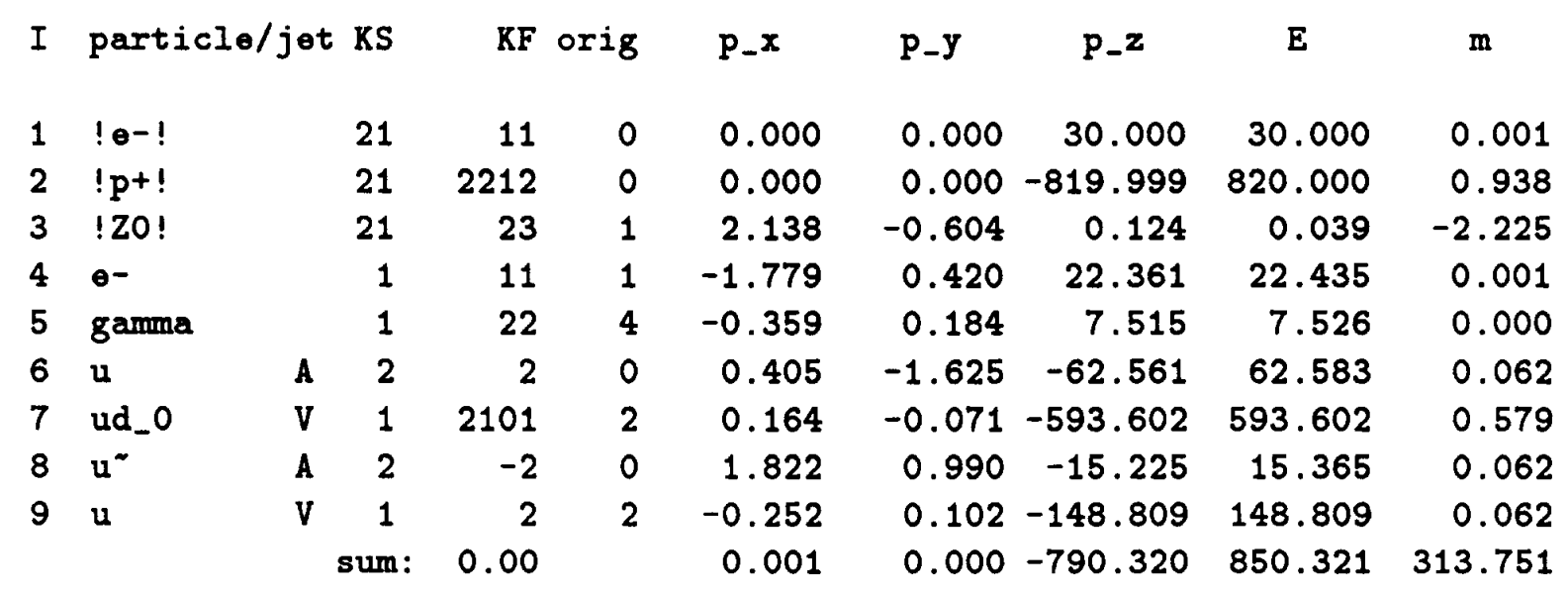

